\title{
Constraint-preserving Sommerfeld conditions for the harmonic Einstein equations
}

\author{
M. C. Babiuc, ${ }^{1}$ H-O. Kreiss, ${ }^{2,3}$ and Jeffrey Winicour ${ }^{1,3}$ \\ ${ }^{1}$ Department of Physics and Astronomy, University of Pittsburgh, Pittsburgh, Pennsylvania 15260, USA \\ ${ }^{2}$ NADA, Royal Institute of Technology, 10044 Stockholm, Sweden \\ ${ }^{3}$ Max-Planck-Institut für Gravitationsphysik, Albert-Einstein-Institut, 4476 Golm, Germany
}

(Received 11 December 2006; published 2 February 2007)

\begin{abstract}
The principle part of Einstein equations in the harmonic gauge consists of a constrained system of 10 curved space wave equations for the components of the space-time metric. A new formulation of constraint-preserving boundary conditions of the Sommerfeld-type for such systems has recently been proposed. We implement these boundary conditions in a nonlinear $3 \mathrm{D}$ evolution code and test their accuracy.
\end{abstract}

DOI: 10.1103/PhysRevD.75.044002

\section{INTRODUCTION}

We present here the implementation and test results of a new formulation of constraint-preserving Sommerfeldtype boundary conditions for Einstein's equations. The well-posedness of the initial-boundary value problem (IBVP) for symmetric hyperbolic systems with maximally dissipative boundary conditions can be established by the energy method [1]. An alternative technique, based on the principle of frozen coefficients, Laplace-Fourier decomposition, and the theory of pseudodifferential operators, can be used to establish well-posedness in a generalized sense even if the boundary conditions are not maximally dissipative or the system is not symmetric hyperbolic [2,3]. This theory has recently been applied to formulate a wellposed, constraint-preserving IBVP for the harmonic Einstein equations with boundary conditions of the Sommerfeld type [4]. In this paper, we show how these new boundary conditions can be implemented in a finitedifference harmonic code in which the Einstein equations are reduced to second order wave equations. The test results presented here show that this new approach has potential value for the computation of gravitational waves in a highly dynamical and nonlinear regime. Although our application here is limited to test problems, we expect these techniques to further the recent progress in the simulation of black holes by harmonic evolution [5-8].

The first well-posed formulation of the IBVP for Einstein's equations was presented in the pioneering work of Friedrich and Nagy [9], based upon a quite different formulation of the Einstein equations. The underlying pseudodifferential theory and how it leads to a constraintpreserving IBVP for the harmonic Einstein equations which is well-posed in a generalized sense is described in [4]. In that work, the details were presented for the linearized Einstein equations but it was explained how the general pseudodifferential theory extends well-posedness to the full nonlinear case. Subject to a certain inequality which is necessary to establish the required estimates, there is considerable freedom in the detailed form of the Sommerfeld-type boundary conditions.
PACS numbers: 04.25.Dm, 04.25.Nx, 04.30.Db, 04.70.Bw

In Sec. II we describe how this new boundary treatment can be implemented in a fully nonlinear code for the simplest choice of the boundary conditions considered in [4]. The resulting scheme is attractive for numerical use. In a previous study [10], constraint-preserving boundary conditions for the harmonic Einstein equations were based upon a combination of Dirichlet and Neumann conditions, which are only marginally dissipative. The description of a traveling wave requires the proper inhomogeneous Dirichlet or Neumann boundary data for the wave to pass through the boundary. However, in numerical simulations, such inhomogeneous Dirichlet or Neumann data can only be prescribed for the signal and the numerical error is reflected by the boundary and accumulates in the grid. Test results show that this can lead to poor performance in the simulation of highly dynamical and nonlinear solutions of Einstein's equations [11,12]. For such computational purposes, it is more advantageous to use a Sommerfeld condition which is strictly dissipative and allows numerical error to leave the grid [13].

The numerical implementation of the boundary conditions, described in Sec. III, is carried out using two distinct approaches. One approach is based upon summation by parts (SBP), which incorporates semidiscrete versions of the conservation laws obeyed by the principle part of the system. The stability of the finite-difference scheme then follows from a discretized energy argument. The second is based upon the embedded boundary method, which is more easily applied to the case of a curved boundary. Theorems regarding the stability of the embedded boundary method for the second order wave equation have been given for the case of Dirichlet and Neumann boundary conditions $[14,15]$. We expect that these theorems can be extended to the strictly dissipative Sommerfeld case.

We compare these two numerical implementations using the standardized AppleswithApples linearized wave, gauge wave [16], and shifted gauge wave [11] tests, modified to include a boundary as documented in [17]. These tests allow a direct comparison of the SBP and embedded boundary methods in a context where the boundary is aligned with the grid. The test results are given in Sec. IV. 


\section{CONSTRAINT-PRESERVING SOMMERFELD BOUNDARY CONDITIONS}

Our results apply to the generalized harmonic formalism including harmonic gauge source terms [18] and constraint adjustments, as described in [11,19]. Generalized harmonic coordinates $x^{\alpha}=\left(t, x^{i}\right)=(t, x, y, z)$ are independent solutions of the curved space scalar wave equation

$$
\square x^{\mu}=\frac{1}{\sqrt{-g}} \partial_{\alpha}\left(\sqrt{-g} g^{\alpha \beta} \partial_{\beta} x^{\mu}\right)=-\hat{\Gamma}^{\mu},
$$

with gauge source terms $\hat{\Gamma}^{\mu}\left(x^{\alpha}, g_{\alpha \beta}\right)$ depending on the coordinates and the metric. In terms of the connection $\Gamma_{\alpha \beta}^{\mu}$, these harmonic conditions take the form

$$
\mathcal{C}^{\mu}:=\Gamma^{\mu}-\hat{\Gamma}^{\mu}=0,
$$

where

$$
\Gamma^{\mu}=g^{\alpha \beta} \Gamma_{\alpha \beta}^{\mu}=-\frac{1}{\sqrt{-g}} \partial_{\alpha} \gamma^{\alpha \mu}
$$

and $\gamma^{\mu \nu}=\sqrt{-g} g^{\mu \nu}$. These $\mathcal{C}^{\mu}$ are the constraints of the harmonic formulation.

Constraint adjustments of the form

$$
A^{\mu \nu}=\mathcal{C}^{\rho} A_{\rho}^{\mu \nu}\left(x^{\alpha}, g^{\alpha \beta}, \partial_{\gamma} g^{\alpha \beta}\right)
$$

can be introduced to modify the reduced system of harmonic equations. These reduced equations then take the form

$$
\tilde{E}^{\mu \nu}:=G^{\mu \nu}-\nabla^{(\mu} \mathcal{C}^{\nu)}+\frac{1}{2} g^{\mu \nu} \nabla_{\alpha} \mathcal{C}^{\alpha}+A^{\mu \nu},
$$

which are equivalent to Einstein equations $G^{\mu \nu}=0$ when the constraints $\mathcal{C}^{\mu}$ are satisfied. When the constraint adjustments and gauge source terms vanish, they reduce to the standard harmonic reduction of the Einstein tensor (see e.g. $[18,20])$,

$$
E^{\mu \nu}:=G^{\mu \nu}-\nabla^{(\mu} \Gamma^{\nu)}+\frac{1}{2} g^{\mu \nu} \nabla_{\alpha} \Gamma^{\alpha}=0 .
$$

The systems (2.5) and (2.6) have the same principle part and they both constitute a constrained system of quasilinear wave equations with a well-posed Cauchy problem. In terms of the metric, these quasilinear wave equations (2.5) can be put in the form

$$
2 \sqrt{-g} \tilde{E}^{\mu \nu}=g^{\alpha \beta} \partial_{\alpha} \partial_{\beta} \gamma^{\mu \nu}+\hat{S}^{\mu \nu}=0,
$$

where $\hat{S}^{\mu \nu}$ are terms which do not contribute to the principle part.

The solutions of the generalized harmonic evolution system (2.5) are solutions of the Einstein equations provided the constraints $\mathcal{C}^{\mu}$ are satisfied. The Bianchi identities, applied to (2.5), imply that $\mathcal{C}^{\mu}$ obeys the homogeneous wave equation

$$
\nabla^{\alpha} \nabla_{\alpha} \mathcal{C}^{\mu}+R_{\nu}^{\mu} \mathcal{C}^{\nu}-2 \nabla_{\nu}\left(\mathcal{C}^{\rho} A_{\rho}^{\mu \nu}\right)=0 .
$$

The well-posedness of the Cauchy problem for (2.8) en- forces the unique solution $\mathcal{C}^{\mu}=0$ in the domain of dependence of the initial Cauchy hypersurface $\mathcal{S}$ provided the Cauchy data $\left.\gamma^{\mu \nu}\right|_{\mathcal{S}}$ and $\left.\partial_{t} \gamma^{\mu \nu}\right|_{\mathcal{S}}$ satisfy $\left.\mathcal{C}^{\mu}\right|_{\mathcal{S}}=\left.\partial_{t} \mathcal{C}^{\mu}\right|_{\mathcal{S}}=$ 0 via (2.5). It is straightforward to show that these initial conditions are satisfied if the data on $\mathcal{S}$ satisfy the Hamiltonian and momentum constraints $G_{\mu}^{t}=0$ and the initial condition $\mathcal{C}^{\mu}=0$.

In order to extend constraint preservation to the IBVP with boundary $\mathcal{B}$ it is sufficient to prescribe boundary conditions for $\gamma^{\mu \nu}$ which imply a maximally dissipative homogeneous boundary condition for $\mathcal{C}^{\mu}$. In $[10,12]$, this was achieved by a combination of Dirichlet and Neumann boundary conditions on the various components of $\gamma^{\mu \nu}$, which then induce a combination of homogeneous Dirichlet and Neumann conditions on the components of $\mathcal{C}^{\mu}$. However, test results showed that Dirichlet and Neumann boundary conditions, which are only borderline dissipative, were considerably less accurate than a strictly dissipative Sommerfeld condition [11]. These test results were based upon exact solutions for which the correct Sommerfeld data were known. Here we consider another approach in which constrained Sommerfeld data may be applied consistently in the absence of an exact solution. This Sommerfeld data for the components of $\gamma^{\mu \nu}$ lead to the homogeneous Dirichlet condition

$$
\left.\mathcal{C}^{\mu}\right|_{\mathcal{S}}=0
$$

on the constraints, which is sufficient to guarantee a constraint-preserving well-posed IBVP. It is possible that a homogeneous Sommerfeld boundary condition on the constraints would lead to better constraint preservation in numerical applications. This requires a second differential order boundary condition on the metric variables for which existing theory gives little guidance. See [21] for a fuller discussion and some promising results.

Well-posedness depends only on the principle part of the quasilinear system (2.7). The pseudodifferential theory implies the principle of frozen coefficients by which wellposedness for the nonlinear problem can be established by treating the metric $g^{\alpha \beta}$ governing the wave operator in (2.7) as a constant. Thus the metric can be transformed by a linear transformation into Minkowski form and without loss of generality it suffices to establish well-posedness for the system of flat space wave equations

$$
\eta^{\alpha \beta} \partial_{\alpha} \partial_{\beta} \gamma^{\mu \nu}=0
$$

subject to the constraints (2.2). In this local Minkowski frame, where $\sqrt{-g}=1$, the constraints reduce to

$$
\mathcal{C}^{\mu}:=-\partial_{\nu} \gamma^{\mu \nu}-\hat{\Gamma}^{\mu}=0 .
$$

We choose our local frame so that the $+x$-direction is the outward normal to $\mathcal{B}$ and the $x^{a}=(t, y, z)$-directions are tangent to $\mathcal{B}$. We write $x^{A}=(y, z)$. The Sommerfeld boundary condition on a scalar field $\Phi$ then takes the form 


$$
\left.\left(\partial_{t}+\partial_{x}\right) \Phi\right|_{\mathcal{B}}=q\left(x^{a}\right),
$$

where $q\left(x^{a}\right)$ represents the prescribed Sommerfeld data. Because the Sommerfeld condition is strictly dissipative it leads to a well-posed IBVP in the case of the scalar wave equation.

Constraint-preserving boundary conditions for the system of linearized Einstein equations (2.10) and (2.11) can be expressed as a hierarchy of Sommerfeld boundary conditions. There are numerous options in this approach [4] and here we consider the mathematically simplest scheme.

First we require the 6 Sommerfeld boundary conditions

$$
\begin{gathered}
\left(\partial_{t}+\partial_{x}\right) \gamma^{A B}=q^{A B}\left(x^{a}\right), \\
\left(\partial_{t}+\partial_{x}\right)\left(\gamma^{t A}-\gamma^{x A}\right)=q^{t A}\left(x^{a}\right)-q^{x A}\left(x^{a}\right), \\
\left(\partial_{t}+\partial_{x}\right)\left(\gamma^{t t}-2 \gamma^{t x}+\gamma^{x x}\right)= \\
q^{t t}\left(x^{a}\right)-2 q^{t x}\left(x^{a}\right) \\
+q^{x x}\left(x^{a}\right),
\end{gathered}
$$

where the $q$ 's are freely prescribed Sommerfeld data. Next, the constraints are used to supply 4 additional boundary conditions in the hierarchical order

$$
\left.\mathcal{C}^{A}\right|_{\mathcal{B}}=-\partial_{t} \gamma^{A t}-\partial_{x} \gamma^{A x}-\partial_{B} \gamma^{A B}-\hat{\Gamma}^{A}\left(x^{a}\right)=0,
$$

$$
\begin{aligned}
\left.\mathcal{C}^{t}\right|_{\mathcal{B}}-\left.\mathcal{C}^{x}\right|_{\mathcal{B}}= & -\partial_{t}\left(\gamma^{t t}-\gamma^{x t}\right)-\partial_{x}\left(\gamma^{x t}-\gamma^{x x}\right) \\
& -\partial_{B}\left(\gamma^{t B}-\gamma^{x B}\right)-\hat{\Gamma}^{t}\left(x^{a}\right)+\hat{\Gamma}^{x}\left(x^{a}\right)=0,
\end{aligned}
$$

$$
\left.\mathcal{C}^{t}\right|_{\mathcal{B}}=-\partial_{t} \gamma^{t t}-\partial_{x} \gamma^{t x}-\partial_{B} \gamma^{t B}-\hat{\Gamma}^{t}\left(x^{a}\right)=0 .
$$

By using (2.13), (2.14), and (2.15), the boundary conditions (2.16), (2.17), and (2.18) can be reexpressed in the Sommerfeld form

$$
\begin{aligned}
\left.\mathcal{C}^{A}\right|_{\mathcal{B}}=-\frac{1}{2}\left(\partial_{t}+\partial_{x}\right)\left(\gamma^{A t}+\gamma^{A x}\right)-\partial_{t}\left(\gamma^{A t}-\gamma^{A x}\right) & -\partial_{B} \gamma^{A B}+\frac{1}{2}\left(q^{t A}-q^{x A}\right)-\hat{\Gamma}^{A}=0, \\
\left.\mathcal{C}^{t}\right|_{\mathcal{B}}-\left.\mathcal{C}^{x}\right|_{\mathcal{B}}= & -\frac{1}{2}\left(\partial_{t}+\partial_{x}\right)\left(\gamma^{t t}-\gamma^{x x}\right) \\
& -\partial_{t}\left(\gamma^{t t}-2 \gamma^{x t}+\gamma^{x x}\right)-\partial_{B}\left(\gamma^{t B}-\gamma^{x B}\right) \\
& +\frac{1}{2}\left(q^{t t}-2 q^{t x}+q^{x x}\right)-\hat{\Gamma}^{t}+\hat{\Gamma}^{x}=0,
\end{aligned}
$$

$$
\begin{aligned}
\left.\mathcal{C}^{t}\right|_{\mathcal{B}}= & -\frac{1}{2}\left(\partial_{t}+\partial_{x}\right)\left(\gamma^{t t}+\gamma^{x x}\right)-\partial_{t}\left(\gamma^{t t}-\gamma^{t x}\right) \\
& +\frac{1}{2}\left(q^{t t}-2 q^{t x}+q^{x x}\right)-\partial_{B} \gamma^{t B}-\hat{\Gamma}^{t}=0 .
\end{aligned}
$$

Equations (2.13), (2.14), (2.15), (2.19), (2.20), and (2.21) form a hierarchical sequence of inhomogeneous Sommerfeld boundary conditions in which the source terms for (2.19), (2.20), and (2.21) are provided by previous members in the hierarchy. In the linearized problem, the boundary conditions (2.13), (2.14), and (2.15), along with compatible initial data that satisfy the constraints, determine unique solutions of the wave equations for $\gamma^{A B}$, $\gamma^{A t}-\gamma^{A x}$, and $\gamma^{t t}-2 \gamma^{t x}+\gamma^{x x}$. These then provide the source terms in (2.19) and (2.20), which determine unique solutions for $\gamma^{A t}+\gamma^{A x}$ and $\gamma^{t t}-\gamma^{x x}$. Finally, the source terms in (2.20) can be determined to provide a unique solution for $\gamma^{t t}+\gamma^{x x}$.

The ten linearly independent Sommerfeld-type boundary conditions (2.13), (2.14), (2.15), (2.19), (2.20), and (2.21) for the components of $\gamma^{\mu \nu}$ give rise to a unique solution of the constrained linearized problem. However, it is important to emphasize the following points:

(1) Proof of the well-posedness of the IBVP requires estimates on the derivatives of the solution at the boundary. For that purpose, it is required to apply the pseudodifferential theory to construct a symmetrizer in Fourier-Laplace space, as described in [4].

(2) The pseudodifferential theory allows wellposedness to be extended locally in time to the nonlinear IBVP for the harmonic Einstein's equations, where the relation $\gamma^{\mu \nu}=\sqrt{-g} g^{\mu \nu}$ converts (2.7) into a quasilinear equation.

(3) Sommerfeld boundary conditions are strictly dissipative but they completely remove all reflections only in highly idealized cases.

(4) The hierarchical structure of the boundary conditions, together with their dissipative property, is a very good heuristic procedure to formulate a stable finite-difference approximation but by itself does not provide a proof of stability.

(5) In the electromagnetic case, our approach gives rise to constraint-preserving Sommerfeld-type boundary conditions on the vector potential which can be given a physical interpretation in terms of the Poynting vector [4]. An analogous interpretation does not exist in the gravitational case. When $q^{A B}$ has a vanishing trace-free part, the boundary condition (2.13) implies that the outgoing null hypersurfaces emanating from the boundary have vanishing shear. This is related to the vanishing of incoming radiation but in a very gauge dependent way. Friedrich and Nagy [9] stress this same caveat concerning their boundary conditions, which specify the Newman-Penrose Weyl component $\Psi_{0}$ associated with the outgoing null hypersurfaces.

(6) In [4], well-posedness of the IBVP was established for the special case where the boundary $x=0$ contains the timelike normal to the initial Cauchy hypersurface $t=0$. More generally, the initial Cauchy hypersurface is given by $T=t-k x$ so that the initial data is different from the initial data at $t=$ 0 . Well-posedness of this more general IBVP can be reduced to well-posedness of the special case by first 
considering a pure Cauchy evolution from $T=0$ to $t=0$. The general case corresponds to a boundary that is "moving" with respect to the Cauchy hypersurface. This introduces a shift term in the wave operator. In the next section, we give the details of how to translate the conditions (2.13), (2.14), (2.15), (2.19), (2.20), and (2.21) into a nonlinear computational scheme for a general metric which is not in the shift-free Minkowski form.

\section{NUMERICAL IMPLEMENTATION}

Our implementation is based upon the Abigel code $[10,12]$, in which the harmonic system (2.7)) is integrated in the first order in time form

$$
\begin{gathered}
\partial_{t} \gamma^{\mu \nu}=T^{\mu \nu}, \\
\partial_{t} T^{\mu \nu}=F^{\mu \nu}\left(\gamma, \partial_{i} \gamma, T, \partial_{i} T\right) .
\end{gathered}
$$

Here $x^{i}=(x, y, z)$ and $\partial_{i}$ denotes spatial derivatives. The code is based upon an explicit, second order accurate finite-difference scheme. Introduction of a spatial grid and finite-difference approximations for the spatial derivatives reduces (3.1) and (3.2) to a large set of ordinary differential equations (method of lines), which are evolved with a fourth order Runge-Kutta integration. Details of the finite-difference approximations are given in [10,12], where the system (3.1) and (3.2) was expressed in flux conservative form and SBP was used to apply the boundary conditions, which enforced the semidiscrete version of the conservation laws obeyed by the principle part The tests in this paper have been carried out with the $\hat{W}$ form of the algorithm described in [12], in which certain nonlinear coefficients are approximated by their averages between grid points. We also add artificial dissipation to (3.1) and (3.2) by the modifications

$$
\begin{aligned}
& \partial_{t} \gamma^{\mu \nu} \rightarrow \partial_{t} \gamma^{\mu \nu}+\epsilon_{\gamma} \mathcal{D}^{4} \gamma^{\mu \nu}, \\
& \partial_{t} T^{\mu \nu} \rightarrow \partial_{t} T^{\mu \nu}+\epsilon_{T} \mathcal{D}^{4} T^{\mu \nu},
\end{aligned}
$$

where $\mathcal{D}^{2}$ is the SBP approximation for the Laplace operator.

The SBP approach requires that the boundary be aligned with the numerical grid. Here we also consider implementation of the boundary conditions by means of the embedded boundary method $[14,15]$, which is applicable even when the boundary is not aligned with the grid.

\section{A. The Sommerfeld boundary data}

We describe the implementation of constraintpreserving Sommerfeld boundary conditions for the fully nonlinear harmonic system (2.5). For purpose of discussion, we locate the boundary $\mathcal{B}$ at $x=$ const with the outer normal in the $+x$-direction. We denote the coordinates intrinsic to the boundary by $x^{a}=(t, y, z)=\left(t, x^{A}\right)$. We introduce an orthonormal tetrad $\left(T^{\mu}, X^{\mu}, Y^{\mu}, Z^{\mu}\right)$, oriented at $\mathcal{B}$ so that $X_{\mu}=\left(0,1 / \sqrt{g^{x x}}, 0,0\right)$ is the unit outward normal and $T^{\mu}=\left(1 / \sqrt{-g_{t t}}, 0,0,0\right)$ is a unit vector in the evolution direction. In addition to the standard requirements of the IBVP that the Cauchy hypersurfaces be spacelike and that the boundary be timelike, we require that $T^{\mu}$ be timelike, i.e. that the evolution be subluminal.

The Sommerfeld data for $\gamma^{\mu \nu}$ consist of

$$
K^{\alpha} \partial_{\alpha} \gamma^{\mu \nu}=q^{\mu \nu},
$$

where $K^{\mu}=T^{\mu}+X^{\mu}$ and $K^{\mu} K_{\mu}=0$. The description of the free and constrained components of $q^{\mu \nu}$ is algebraically more complicated than for the Minkowski case in Sec. II. For this reason, it is useful to introduce the projection operators

$$
M_{\mu}^{\nu}=\delta_{\mu}^{\nu}+T_{\mu} T^{\nu}-X_{\mu} X^{\nu}=Y_{\mu} Y^{\nu}+Z_{\mu} Z^{\nu},
$$

which projects vectors into the $\left(Y^{\mu}, Z^{\mu}\right)$ spatial-plane tangent to the boundary, and

$$
P_{\mu}^{\nu}=\delta_{\mu}^{\nu}+\frac{1}{2} L_{\mu} K^{\nu},
$$

where $L^{\mu}=T^{\mu}-X^{\mu}$ with $K^{\mu} L_{\mu}=-2$, which projects vectors into the null 3-space spanned by $\left(L^{\mu}, Y^{\mu}, Z^{\mu}\right)$. We have $P_{\mu}^{\nu} K^{\mu}=0$ and $P_{\mu}^{\nu} L_{\nu}=0$, i.e. $P_{\mu}^{\nu}$ projects forms $W_{\mu}$ into the subspace orthogonal to $K_{\mu}$. We have

$$
P_{\mu}^{\nu}=M_{\mu}^{\nu}-\frac{1}{2} K_{\mu} L^{\nu}
$$

The freely prescribed Sommerfeld data corresponding to (2.13), (2.14), and (2.15) consist of the projection

$$
Q^{\mu \nu}=P_{\alpha}^{\mu} P_{\beta}^{\nu} q^{\alpha \beta} \text {. }
$$

The constrained data consist of $Q^{\mu}=P_{\alpha}^{\mu} L_{\beta} q^{\alpha \beta}$, analogous to (2.19) and (2.20); and $Q=L_{\alpha} L_{\beta} q^{\alpha \beta}$, analogous to (2.21). In order to determine the constrained data, we use the identity

$$
\partial_{\alpha} \gamma^{\alpha \nu}=-\frac{1}{2} L_{\alpha} q^{\alpha \nu}+P_{\alpha}^{\rho} \partial_{\rho} \gamma^{\alpha \nu} .
$$

The constraints then imply

$$
-\sqrt{-g} \hat{\Gamma}^{\nu}=-\frac{1}{2} L_{\alpha} q^{\alpha \nu}+P_{\alpha}^{\rho} \partial_{\rho} \gamma^{\alpha \nu}
$$

so that

$$
Q^{\mu}=2 P_{\alpha}^{\mu} P_{\beta}^{\rho} \partial_{\rho} \gamma^{\alpha \beta}+2 \sqrt{-g} P_{\alpha}^{\mu} \hat{\Gamma}^{\alpha}
$$

and

$$
Q=2 L_{\alpha} P_{\beta}^{\rho} \partial_{\rho} \gamma^{\alpha \beta}+2 \sqrt{-g} L_{\alpha} \hat{\Gamma}^{\alpha} .
$$

The full set of Sommerfeld data consist of

$$
q^{\mu \nu}=Q^{\mu \nu}-Q^{(\mu} K^{\nu)}+\frac{1}{4} Q K^{\mu} K^{\nu} .
$$

As in (2.19), (2.20), and (2.21), the derivatives normal to the boundary occurring in (3.12) and (3.13) can be eliminated via the identities 


$$
\begin{aligned}
P_{\alpha}^{\mu} P_{\beta}^{\rho} \partial_{\rho} \gamma^{\alpha \beta}= & X_{\alpha} Q^{\mu \alpha}-P_{\alpha}^{\mu} K_{\beta} T^{\rho} \partial_{\rho} \gamma^{\alpha \beta} \\
& +P_{\alpha}^{\mu} M_{\beta}^{\rho} \partial_{\rho} \gamma^{\alpha \beta}
\end{aligned}
$$

and

$$
\begin{aligned}
L_{\alpha} P_{\beta}^{\rho} \partial_{\rho} \gamma^{\alpha \beta}= & \frac{1}{2} K_{\alpha} Q^{\alpha}-L_{\alpha} K_{\beta} T^{\rho} \partial_{\rho} \gamma^{\alpha \beta} \\
& +L_{\alpha} M_{\beta}^{\rho} \partial_{\rho} \gamma^{\alpha \beta} .
\end{aligned}
$$

Combined with (3.12) and (3.13), these equations determine the constrained boundary data $Q^{\mu}$ and $Q$ in terms of quantities intrinsic to the boundary,

$$
\begin{aligned}
Q^{\mu}= & 2\left(\frac{1}{2} K_{\alpha} Q^{\mu \alpha}-P_{\alpha}^{\mu} K_{\beta} T^{\rho} \partial_{\rho} \gamma^{\alpha \beta}+P_{\alpha}^{\mu} M_{\beta}^{\rho} \partial_{\rho} \gamma^{\alpha \beta}\right. \\
& \left.+\sqrt{-g} P_{\alpha}^{\mu} \hat{\Gamma}^{\alpha}\right), \\
Q= & 2\left(\frac{1}{2} K_{\alpha} Q^{\alpha}-L_{\alpha} K_{\beta} T^{\rho} \partial_{\rho} \gamma^{\alpha \beta}+L_{\alpha} M_{\beta}^{\rho} \partial_{\rho} \gamma^{\alpha \beta}\right. \\
& \left.+\sqrt{-g} L_{\alpha} \hat{\Gamma}^{\alpha}\right) .
\end{aligned}
$$

\section{B. The boundary update algorithm}

In Sec. III A, we have described how the boundary values of $\gamma^{\mu \nu}, T^{\mu \nu}=\partial_{t} \gamma^{\mu \nu}$ and the free Sommerfeld data $Q^{\mu \nu}$ at time $t$ determine the full Sommerfeld data $q^{\mu \nu}$ at time $t$. We now consider how these quantities are updated. The update of the boundary values of $\gamma^{\mu \nu}$ to the next time step $t+\Delta t$ is straightforward via (3.1)). The update of $T^{\mu \nu}$ is more complicated. Here we use two distinct algorithms, the SBP algorithm and the embedded boundary algorithm, for updating the boundary values of $T^{\mu \nu}$. This provides two competitive updated algorithms, which are compared in test problems in Sec. IV.

SBP algorithm. This is the algorithm described in [12]. The evolution equation (3.2) is applied at the boundary points to update $T^{\mu \nu}$, with field values at the resulting ghost points eliminated by the boundary condition in a manner that enforces discrete conservation laws. Full details for the cases of Dirichlet, Neumann, and Sommerfeld boundary conditions are given in [12]. The only new ingredient here is the use of constraint-preserving Sommerfeld conditions. However, because the constrained Sommerfeld data $Q^{\mu}$ and $Q$ can be updated after the update of $\gamma^{\mu \nu}$ and $T^{\mu \nu}$, there is no essential change in the numerical algorithm.

Embedded boundary algorithm. The SBP algorithm uses the fact that the boundary is aligned with the grid, so that boundary points are grid points. In the case of a spherical boundary and a Cartesian grid, this is generally not the case unless multiblock techniques are used. An alternative approach for treating a curved boundary with a Cartesian grid is the embedded boundary method, in which field values at ghost points are updated from interpolations using the boundary data, as opposed to applying the evolution equation.
Assume that we are given the values of $\gamma^{\mu \nu}$ and $T^{\mu \nu}$ at time $t$ and the free data $Q^{\mu \nu}$ at all times. As above, this determines the full constrained Sommerfeld data $q^{\mu \nu}$ at time $t$. After updating the boundary values of $\gamma^{\mu \nu}$ to time $t+\Delta t$, we update the boundary values $T^{\mu \nu}$ using

$$
q^{\mu \nu}=K^{\alpha} \partial_{\alpha} \gamma^{\mu \nu}=K^{t} T^{\mu \nu}+K^{i} \partial_{i} \gamma^{\mu \nu},
$$

where the spatial derivatives $K^{i} \partial_{i} \gamma^{\mu \nu}$ at the boundary are determined to be an interpolation scheme (see below). When all components of the Sommerfeld data $q^{\mu \nu}$ are supplied analytically, this provides updated boundary values for $T^{\mu \nu}$, which we refer to as the analytic version of the embedded boundary algorithm.

In the absence of an analytic solution, only the unconstrained components of the Sommerfeld data $Q^{\mu \nu}$ can be prescribed freely. The update of $T^{\mu \nu}$ is then first carried out for those components determined by the free data, i.e.

$$
\tau^{\mu \nu}=P_{\alpha}^{\mu} P_{\beta}^{\nu} T^{\alpha \beta},
$$

for which (3.19) gives

$$
Q^{\mu \nu}=K^{t} \tau^{\mu \nu}+P_{\alpha}^{\mu} P_{\beta}^{\nu} K^{i} \partial_{i} \gamma^{\alpha \beta} .
$$

In order to update the boundary values of the remaining components of $T^{\mu \nu}$, we must first update the constrained Sommerfeld data $Q^{\mu}$ and $Q$ by expressing all time derivatives on the right-hand sides of (3.17) and (3.18) in terms of previously updated quantities. From (3.17), we obtain

$$
\begin{aligned}
Q^{\mu}= & 2\left(\frac{1}{2} K_{\alpha} Q^{\mu \alpha}-\frac{1}{\sqrt{-g_{t t}}} K_{\alpha} \tau^{\mu \alpha}+M_{\alpha}^{t} \tau^{\mu \alpha}\right. \\
& \left.+P_{\alpha}^{\mu} M_{\beta}^{i} \partial_{i} \gamma^{\alpha \beta}+\sqrt{-g} P_{\alpha}^{\mu} \hat{\Gamma}^{\alpha}\right) .
\end{aligned}
$$

This determines

$$
\tau^{\mu}=P_{\alpha}^{\mu} L_{\beta} T^{\alpha \beta}
$$

through

$$
Q^{\mu}=K^{t} \tau^{\mu}+P_{\alpha}^{\mu} L_{\beta} K^{i} \partial_{i} \gamma^{\alpha \beta} .
$$

Similarly, from (3.18), we next obtain

$$
\begin{aligned}
Q= & 2\left(\frac{1}{2} K_{\alpha} Q^{\alpha}-\frac{1}{\sqrt{-g_{t t}}} K_{\alpha} \tau^{\alpha}+M_{\alpha}^{t} \tau^{\alpha}+L_{\alpha} M_{\beta}^{i} \partial_{i} \gamma^{\alpha \beta}\right. \\
& \left.+\sqrt{-g} L_{\alpha} \hat{\Gamma}^{\alpha}\right) .
\end{aligned}
$$

This determines

$$
\tau=L_{\alpha} L_{\beta} T^{\alpha \beta}
$$

through

$$
Q=K^{t} \tau+L_{\alpha} L_{\beta} K^{i} \partial_{i} \gamma^{\alpha \beta} .
$$

These pieces allow us to construct

$$
T^{\mu \nu}=\tau^{\mu \nu}-\tau^{(\mu} K^{\nu)}+\frac{1}{4} \tau K^{\mu} K^{\nu} .
$$


In carrying out this update of $T^{\mu \nu}$ it is necessary to compute the boundary values of the spatial derivatives $K^{i} \partial_{i} \gamma^{\mu \nu}$ which appear in (3.19). This is accomplished by an interpolation scheme patterned after the embedded boundary treatment for a Neumann condition given in [15]. In this scheme, the value of $K^{i} \partial_{i} \gamma^{\mu \nu}$ at a boundary point $B$, with grid values $x_{B}^{i}=\left(I_{B}, J_{B}, K_{B}\right) h$, is obtained from the 1-dimensional Lagrange polynomial for $\gamma^{\mu \nu}$ determined by $B$ and $M$ interior points, $C_{m}(1 \leq m \leq$ $M$ ), lying along the curve

$$
x^{i}=x_{B}^{i}+\frac{K^{i}}{K^{x}}\left(x-x_{B}\right)
$$

normal to the boundary. We choose $\left(x_{B}-x_{C_{m}}\right)=m h$ so that the points $C_{m}$ lie in $(y, z)$ planes through the $x$-grid. The values of $\gamma^{\mu \nu}$ at the points $C_{m}$ are obtained by $2 \mathrm{D}$ interpolations in those planes. We use a $2 \mathrm{D}$ Lagrange interpolant based upon a stencil of grid points which is symmetrical about the point $\left(y_{B}, z_{B}\right)$. In order to avoid extrapolation, the size of the stencil has to be adjusted to the size of $K^{i} / K^{x}$. For all 2D test cases considered in Sec. IV, it suffices to use a $3 \times 3$ (or larger) stencil in the planes determined by $C_{m}$.

Note that this scheme differs from the straightforward computation of $K^{i} \partial_{i} \gamma^{\mu \nu}$ by centered differencing in the $y$ and $z$ directions and one-sided differencing in the $x$ direction, which would give a qualitatively different and less accurate approximation. However, in the simple case where $K^{y}=K^{z}=0$, this interpolation scheme does reduce to approximating $K^{i} \partial_{i} \gamma^{\mu \nu}=K^{x} \partial_{x} \gamma^{\mu \nu}$ by a onesided finite difference at $B$. For a second order accurate scheme, only 3 points $C_{m}(M=3)$ are necessary to construct the Lagrange polynomial approximating (3.29). However, with a second order accurate interior evolution algorithm, the error would then be largest at the boundary because of the use of a 1-sided derivative. On the other hand, it is simple to use more points if higher accuracy at the boundary is desired. In this respect, the embedded boundary algorithm is more flexible than the SBP algorithm, for which the order of accuracy of the boundary algorithm is coupled with the order of accuracy of the interior algorithm. In the embedded case, for any internal accuracy, the order of accuracy of the boundary condition can be made high as desired at little additional computational expense. In the tests results shown in the next section, we use $M=5$. We also add a corrector step to the boundary update algorithm to improve accuracy.

\section{TESTS}

We conduct three boundary tests which have been proposed for the AppleswithApples (AwA) test suite [17].
These tests extend the original linearized wave, gauge wave, and shifted gauge wave tests with periodic boundaries, i.e. a 3-torus $T^{3}$ without boundary, to include nontrivial boundaries by opening up the $x$-axis of the 3-torus to form a manifold with smooth $T^{2}$ boundaries at $x= \pm .5$. In this way, the tests avoid the complication of sharp boundary points. The corresponding metrics are

(i) Linearized wave:

$$
d s^{2}=-d t^{2}+d x^{2}+(1+H) d y^{2}+(1-H) d z^{2},
$$

(ii) Gauge wave:

$$
d s^{2}=(1-H)\left(-d t^{2}+d x^{2}\right)+d y^{2}+d z^{2},
$$

(iii) Shifted gauge wave:

$$
\begin{aligned}
d s^{2}= & -d t^{2}+d x^{2}+d y^{2}+d z^{2} \\
& +H k_{\alpha} k_{\beta} d x^{\alpha} d x^{\beta},
\end{aligned}
$$

where in all cases

$$
H=H(x-t)=A \sin \left(\frac{2 \pi(x-t)}{d}\right),
$$

and

$$
k_{\alpha}=\partial_{\alpha}(x-t)=(-1,1,0,0) .
$$

These metrics describe sinusoidal traveling waves of amplitude $A$ propagating along the $x$-axis. Two-dimensional features are tested by rotating the coordinates according to

$$
x=\frac{1}{\sqrt{2}}\left(x^{\prime}-y^{\prime}\right), \quad y=\frac{1}{\sqrt{2}}\left(x^{\prime}+y^{\prime}\right)
$$

which produces a wave propagating along the diagonal.

The linearized wave test is run with an amplitude $A=$ $10^{-8}$. It is most efficient for revealing problems arising from nonlinearity to run the gauge wave and shifted gauge wave tests with amplitude $A=0.5$. In some cases we also run with smaller amplitudes in the range $A=.01$ to $A=.1$ (the original AwA specifications) to reveal the emergence of nonlinear features. In all other respects, we retain the original AwA specifications:

(i) Wavelength: $d=1$ in the 1D simulation and $d^{\prime}=$ $1 / \sqrt{2}$ in the $2 \mathrm{D}$ simulation.

(ii) Simulation domain:

$$
\begin{array}{cccc}
\text { 1D: } x \in[-0.5,+0.5], & y=0, & z=0, & d=1 \\
\text { diagonal: } x \epsilon[-0.5,+0.5], & y=\epsilon[-0.5,+0.5], & z=0, & d^{\prime}=\sqrt{2}
\end{array}
$$


(iii) Grid: $\quad x_{n}=-0.5+n d x, \quad n=0,1 \ldots 50 \rho, \quad d x=$ $d y=d z=1 /(50 \rho), \quad \rho=1,2,4$

(iv) Time step: $d t=d x / 4=0.005 / \rho$.

The grids have $N=50 \rho=(50,100,200)$ zones. (At least 50 zones are required to lead to reasonable simulations for more than 10 crossing times.) The $1 \mathrm{D}$ tests are carried out for $t=1000$ crossing times, i.e. $2 \times 10^{5} \rho$ time steps, and the $2 \mathrm{D}$ tests for 100 crossing times.

As an example of how the Sommerfeld boundary data is prescribed, consider the 1D shifted gauge wave. The Sommerfeld operator at the right boundary $x=+.5$ is given by $K^{\alpha} \partial_{\alpha}=\sqrt{1-H}\left(\partial_{t}+\partial_{x}\right)$ and the corresponding Sommerfeld data vanishes $\left(q^{\mu \nu}=0\right)$. At the left boundary $x=-.5$, where the outward normal is in the $(-x)$ direction, the Sommerfeld operator is

$$
K^{\alpha} \partial_{\alpha}=\left(T^{\alpha}-X^{\alpha}\right) \partial_{\alpha}=\frac{1+H}{\sqrt{1-H}} \partial_{t}-\sqrt{1-H} \partial_{x}
$$

and the resulting nonvanishing components of Sommerfeld data are

$$
q^{t t}=q^{t x}=q^{x x}=-\frac{2}{\sqrt{1-H}} \partial_{t} H(x-t) .
$$

With this inhomogeneous Sommerfeld data, the wave enters through the boundary at $x=-.5$, propagates across the grid, and exits through the boundary at $x=+.5$.

At the left boundary $x=-.5$, the inward null direction $L_{\mu}=T_{\mu}+X_{\mu}$, which enters the projection operator (3.7), satisfies $L_{\mu} q^{\mu \nu}=0$. Consequently, the nonvanishing components of the free data $Q^{\mu \nu}$ are also given by (4.8) and the constrained components vanish, i.e. $Q^{\mu}=Q=0$. Of course, in a simulation using the constraint-preserving Sommerfeld algorithm, numerical error gives rise to nonvanishing values for $Q^{\mu}$ and $Q$. Thus it makes a difference at the numerical level whether $Q^{\mu}$ and $Q$ are given their analytic values (zero) or their values produced by the constraint-preserving algorithm. These alternatives will be compared in carrying out the tests to provide a measure of the efficacy of the constraint-preserving algorithm.

Thus for each of the two boundary algorithms (SBP and embedded boundary) we run the tests (i) with all 10 components of Sommerfeld data $q^{\mu \nu}$ provided by the analytic solution and (ii) with the 6 free components of Sommerfeld data $Q^{\mu \nu}$ provided by the analytic solution and the remaining components $Q^{\mu}$ and $Q$ provided by the constraint-preserving algorithm. We distinguish the corresponding tests results by the labels ASBP and AEMB, respectively, for the SBP and embedded algorithms with fully analytic data; and CSBP and CEMB for the corresponding tests with constraint-preserving data.

In the 1D gauge wave tests, the curves (3.29) normal to the boundary pass through the grid points on the $x$-axis, so that the interpolations required for the embedded boundary algorithm are trivial. The 2D gauge wave and shifted gauge wave both provide a nontrivial test of the interpolation scheme. For the case of the shifted gauge wave, the curves (3.29) at the boundaries $x_{B}= \pm .5$ are given by

$$
y=y_{B}+\frac{K^{y}}{K^{x}}\left(x-x_{B}\right) .
$$

Harmonic gauge forcing terms were not found to be effective in the boundary-free gauge wave tests and we have not included them in the present tests. (Gauge forcing is important in spacetimes where harmonic coordinates become pathological, e.g. the standard $t$-coordinate in Schwarzschild spacetime is harmonic but singular at the horizon.) Constraint adjustments were not necessary except in trying to stabilize the constrained SBP shifted gauge wave runs.

We use the $\ell_{\infty}$ norm to measure the error

$$
\mathcal{E}(\Phi)=\left\|\Phi_{\rho}-\Phi_{\text {ana }}\right\|_{\infty}
$$

in a grid function $\Phi_{\rho}$ with known analytic value $\Phi_{\text {ana }}$. We measure the convergence rate at time $t$

$$
r(t)=\log _{2}\left(\frac{\left\|\Phi_{2}-\Phi_{\text {ana }}\right\|_{\infty}}{\left\|\Phi_{4}-\Phi_{\text {ana }}\right\|_{\infty}}\right)
$$

using the $\rho=2$ and $\rho=4$ grids $(N=100$ and $N=200)$. (The $\rho=1$ grid is not very accurate and is mainly used for debugging.) For convergence studies it is also useful to graph the rescaled error

$$
\mathcal{E}_{\rho}=\frac{\rho^{2}}{16}\left\|\Phi_{\rho}-\Phi_{\text {ana }}\right\|_{\infty}
$$

which is normalized to the $\rho=4$ grid.

\section{A. Linearized wave tests}

Table I shows the convergence rates of the error in $g_{y y}$ for the 1D linearized wave, measured at $t=10$ and $t=50$ crossing times. Clean second order convergence is maintained for all four algorithms, irrespective of whether the complete Sommerfeld data is supplied from the analytic solution (ASBP and AEMB), or whether it is constrained (CSBP and CEMB). At 1000 crossing times, the four algorithms continue to give excellent agreement with the analytic solution. The graphs in Fig. 1 show excellent phase agreement and a small difference in amplitude at $t=$ 1000 in the comparison between the analytic solution for $g_{y y}(x)$ and the CEMB and CSBP results. On the scale of

TABLE I. Convergence rates of $\mathcal{E}\left(g_{y y}\right)$ for the 1D linearized wave test, amplitude $A=10^{-8}$.

\begin{tabular}{lcc}
\hline \hline ALGORITHM & $t=10$ & $t=50$ \\
\hline AEMB & 2.04 & 2.05 \\
ASBP & 1.99 & 1.93 \\
CEMB & 2.04 & 1.97 \\
CSBP & 1.99 & 2.03 \\
\hline \hline
\end{tabular}




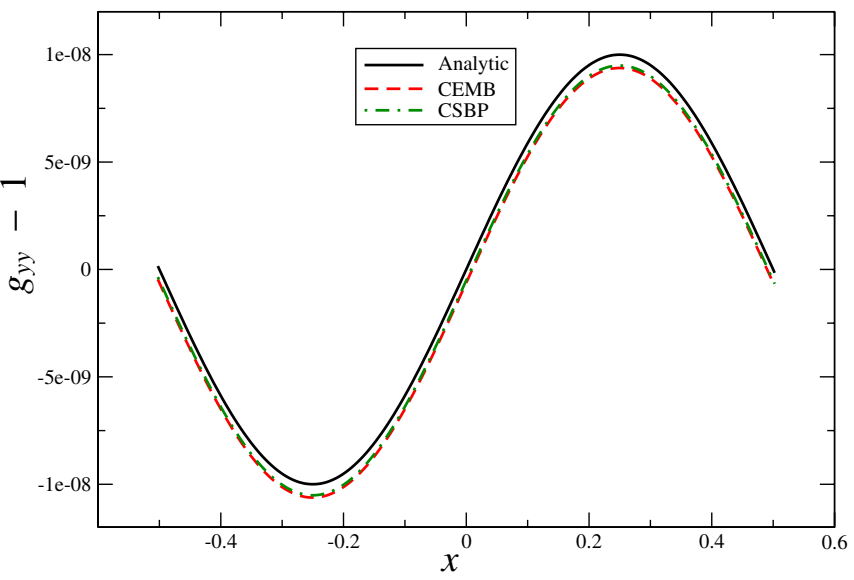

FIG. 1 (color online). The graphs compare the performance of the CEMB and CSBP algorithms for the 1D linearized wave test on the $\rho=4$ grid with the analytic solution. The CEMB and CSBP snapshots of $g_{y y}(x)-1$, shown at $t=1000$, are indistinguishable and show only a small amplitude discrepancy with the analytic solution.

Fig. 1, the graphs for the AEMB and ASBP algorithms are indistinguishable from the analytic solution.

Table II shows the convergence rates of the error in $g_{y y}$ for the 2D linearized wave test, measured at $t=1, t=5$, and $t=10$. Second order convergence is cleanly maintained for the AEMB and ASBP algorithms. The convergence rates of the CEMB and CSBP show some deterioration from second order at $t=10$ as the truncation error from the boundary algorithms accumulates. Nevertheless, at $t=100$, the $2 \mathrm{D}$ results for all the algo-

TABLE II. Convergence rates of $\mathcal{E}\left(g_{y y}\right)$ for the 2D linearized wave test.

\begin{tabular}{lccc}
\hline \hline ALGORITHM & $t=1$ & $t=5$ & $t=10$ \\
\hline AEMB & 1.98 & 2.00 & 1.97 \\
ASBP & 1.96 & 1.93 & 1.92 \\
CEMB & 1.98 & 1.94 & 1.62 \\
CSBP & 1.94 & 1.89 & 1.77 \\
\hline \hline
\end{tabular}

TABLE III. Convergence rates of $\mathcal{E}\left(g_{x x}\right)$ for the $1 \mathrm{D}$ gauge wave test.

\begin{tabular}{lcc}
\hline \hline ALGORITHM & $t=10$ & $t=50$ \\
\hline AEMB & 1.97 & 1.87 \\
ASBP & 2.00 & 2.00 \\
CEMB & 1.97 & 1.87 \\
CSBP & 1.98 & 1.95 \\
\hline \hline
\end{tabular}

rithms remain in excellent agreement with the analytic solution. The projection operators used in the constrained CSBP and CEMB algorithms introduce the small errors shown in Fig. 2, where snapshots of $g_{y y}-1$ are graphed for $y=0$ and $t=100$, using the $\rho=4$ grid. Overall, the linearized wave tests show that both the SBP and embedded algorithms give excellent results for either fully analytic or constrained Sommerfeld boundary conditions.

\section{B. Gauge wave tests}

For the 1D gauge wave tests, Table III shows the convergence rates of the SBP and embedded algorithms, measured at $t=10$ and $t=50$ crossing times. Second order convergence at $t=10$ is clean in both cases. At $t=50$, the convergence rates of the embedded algorithms show slight deterioration, for the reasons explained below.

All the algorithms remain stable for 1000 crossing times for the 1D runs on the $\rho=4$ grid, without use of constraint adjustments or artificial dissipation except for a small amount of dissipation (3.4), with $\epsilon_{T}=0.01$, for the constrained CSBP algorithm. Figure 3 shows the long term performance of the ASBP and AEMB algorithms. Both maintain excellent accuracy for 1000 crossing times. For the ASBP algorithm there is negligible error growth. For the embedded AEMB algorithm, there is long wavelength error corresponding to the harmonic instability [13]

$$
d s_{\lambda}^{2}=e^{\lambda t}(1-H)\left(-d t^{2}+d x^{2}\right)+d y^{2}+d z^{2}
$$

of the gauge wave spacetime (4.2). For any value of $\lambda$, (4.13) is a flat metric which obeys the harmonic constraints. As depicted in Fig. 3, the resulting profile of the
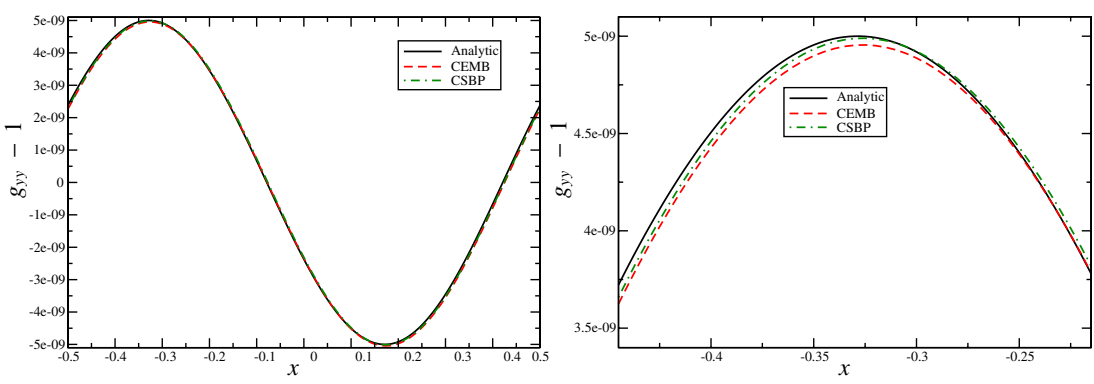

FIG. 2 (color online). Snapshots of $g_{y y}(x)-1$ for the 2D linear wave tests obtained at $t=100$, setting $y=0$. On the left plot, the snapshots of $g_{y y}-1$ for the CEMB and CSBP algorithms are compared with the analytic solution. On this scale the errors are almost imperceptible and a zoom is given in the right plot. 

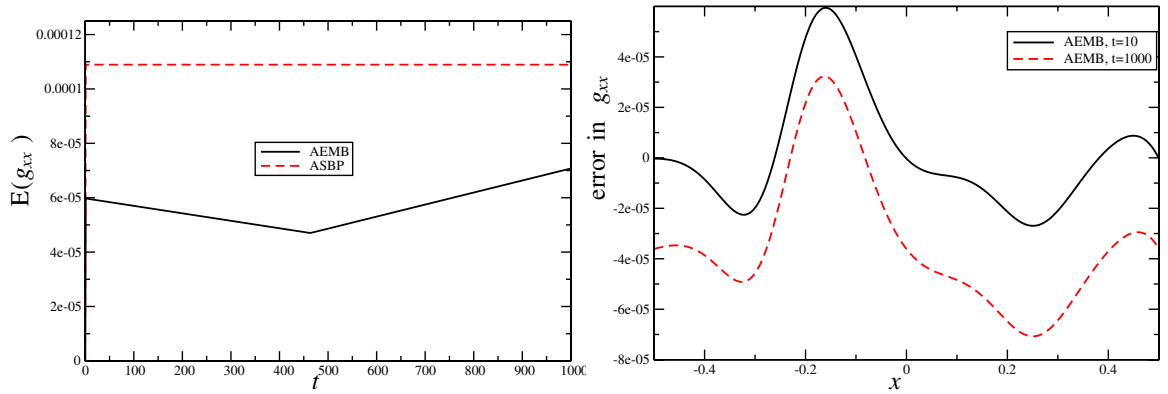

FIG. 3 (color online). The performance of the analytic SBP and embedded algorithms for the 1D gauge wave test. On the left, the error norm $\mathcal{E}\left(g_{x x}\right)$ is plotted vs $t$. On the right, snapshots of the error in $g_{x x}$ for the AEMB algorithm are shown at $t=10$ and $t=1000$. This error is long wavelength, consisting of two peaks. The positive peak dominates in the beginning, but an overall drop in the profile causes the error to pass through a minimum and then to grow again as the negative peak dominates. This behavior is due to the long wavelength instability in the gauge wave spacetime.

AEMB error contains two peaks. The positive peak dominates in the beginning, but there is an overall drop in the waveform, due to the instability, which causes the error to pass through a minimum and then grow again as the negative peak dominates. The SBP algorithm is designed to suppress this instability by means of discrete conservation laws for the principle part of the evolution equations. The embedded algorithm excites the instability although at a fairly innocuous level.

Similar 1D results hold for the constrained algorithms CSBP and CEMB, as shown in Fig. 4. The use of a 5-point $(M=5)$ Lagrange polynomial is essential for the good performance of the constrained algorithms. Figure 5 shows the rapid error growth which would result from the use of 3 or 4 points, again arising from excitation of the long wavelength instability (4.13).

The convergence rates for the $2 \mathrm{D}$ gauge wave tests shown in Table IV indicate clean second order convergence up to $t=10$. The graphs of the error in Fig. 6 show that the analytic SBP and embedded algorithms maintain excellent accuracy up to $t=100$. However, the constrained algo-

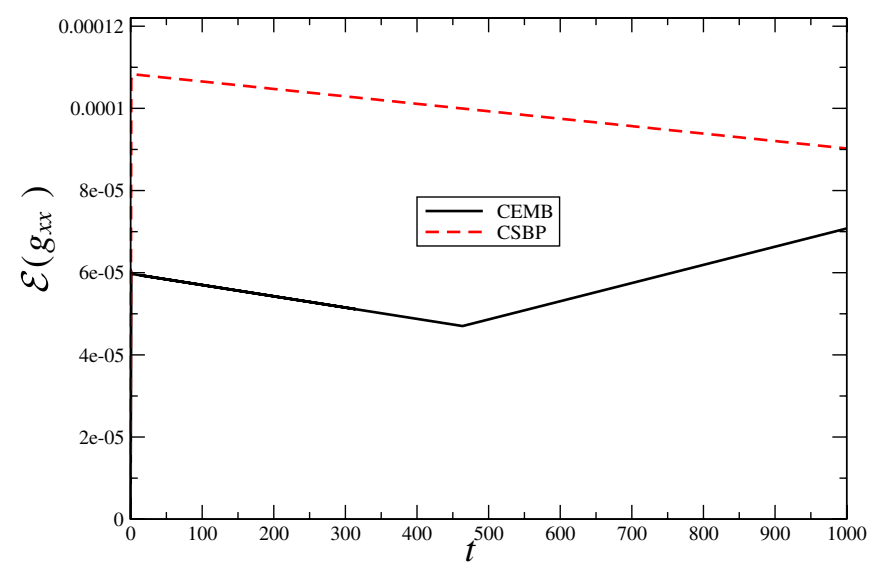

FIG. 4 (color online). Plot of the error norm $\mathcal{E}\left(g_{x x}\right)$ vs $t$ for the 1D gauge wave simulations with the constrained SBP and embedded algorithms, obtained with the $\rho=4$ grid. rithms excite the long wavelength instability (4.13) at $t \approx$ 55 for CSBP and $t \approx 60$ for CEMB. Neither numerical dissipation nor constraint adjustment lead to significant improvement. Higher order accuracy of the boundary condition also does not seem to help.

\section{Shifted gauge wave tests}

For the 1D shifted gauge wave tests, Table V shows the convergence rates of the SBP and embedded algorithms measured at $t=10$ and $t=50$ crossing times. Second order convergence is fairly clean at $t=10$, with some drifting of the convergence rates evident at $t=50$.

A long wavelength instability also exists in the shifted gauge wave spacetime (4.3) [11]

$$
\begin{aligned}
d s_{\lambda}^{2}= & -d t^{2}+d x^{2}+d y^{2}+d z^{2} \\
& +\left(H-1+e^{\lambda \hat{t}}\right) k_{\alpha} k_{\beta} d x^{\alpha} d x^{\beta},
\end{aligned}
$$

where

$$
\hat{t}=t-\frac{A d}{4 \pi} \cos \left(\frac{2 \pi(x-t)}{d}\right) .
$$

Although this metric does not solve Einstein's equations, for any value of $\lambda$ it satisfies the standard harmonic form (2.6) of the reduced Einstein equations, i.e. the equations governing numerical evolution without constraint adjustment. This instability is the major source of error. Figure 7 exhibits the long term performance for runs with the analytic SBP and embedded algorithms. They maintain excellent accuracy for 1000 crossing times, although the snapshots show that the embedded algorithm has produced a low level excitation of the instability.

The larger boundary errors of the constrained algorithms lead to stronger excitation of the long wavelength instability. Without constraint adjustment, the runs on the $\rho=4$ grid crash at about $t \approx 88$ for the CSBP algorithm and $t \approx$ 103 for the CEMB algorithm. Numerical dissipation was necessary in the CEMB run but had insignificant effect on controlling the long wavelength instability in either of the 

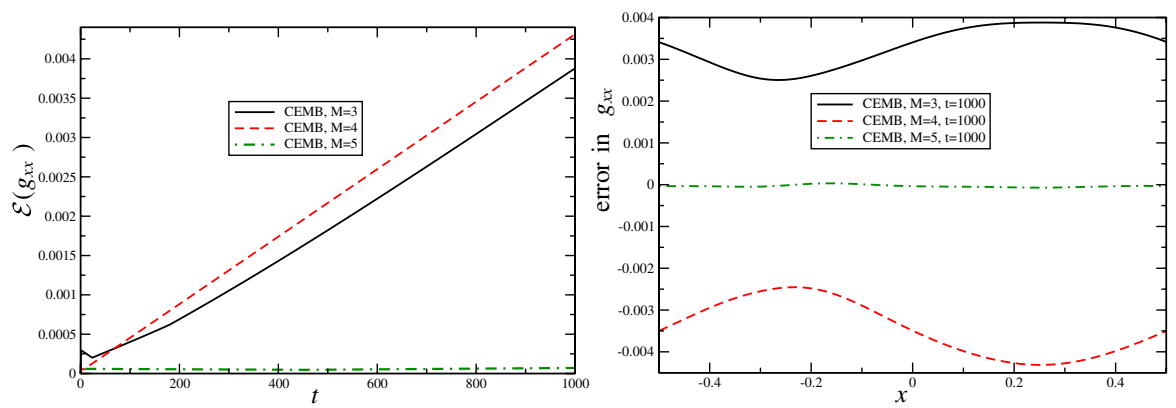

FIG. 5 (color online). On the left, the error norm $\mathcal{E}\left(g_{x x}\right)$ is plotted vs $t$ for the $1 \mathrm{D}$ gauge wave test using the CEMB algorithm with Lagrange polynomials based upon $M=3,4$, and 5 points. Only for $M=5$ is there no long term error growth. On the right, the snapshots of the error in $g_{x x}$ at $t=1000$ show the long wavelength mode which is excited in the $M=3$ and 4 cases.

TABLE IV. Convergence rates of $\mathcal{E}\left(g_{x x}\right)$ for the 2D gauge wave test.

\begin{tabular}{lccc}
\hline \hline ALGORITHM & $t=1$ & $t=5$ & $t=10$ \\
\hline AEMB & 2.02 & 2.03 & 2.03 \\
ASBP & 2.02 & 2.03 & 2.02 \\
CEMB & 2.02 & 2.01 & 2.02 \\
CSBP & 2.02 & 2.01 & 2.03 \\
\hline \hline
\end{tabular}

constrained algorithms. However, constraint adjustment was moderately effective. Of the various adjustments of the form (2.4) which were considered in [11], the longest constrained runs were obtained with the choice

$$
A^{\mu \nu}=\frac{b \mathcal{C}^{\alpha} \nabla_{\alpha} t}{e_{\rho \sigma} \mathcal{C}^{\rho} \mathcal{C}^{\sigma}} \mathcal{C}^{\mu} \mathcal{C}^{\nu}, \quad b>0
$$

Here

$$
e_{\rho \sigma}=g_{\rho \sigma}-\frac{2}{g^{t t}}\left(\nabla_{\rho} t\right) \nabla_{\sigma} t
$$

is the natural metric of signature $(++++)$ associated with the Cauchy slicing. This adjustment with $b=1$ extended the CSBP run to $t \approx 155$ and the CEMB run to $t \approx$ 183 crossing times, as indicated in Fig. 8. The response to constraint adjustment is evidence of the constraint violat- ing origin of the analytic instability. The instability is also of nonlinear origin, which can be seen from the comparison with the runs of lower amplitude shown in Fig. 9. With lower amplitude, the discrete conservation laws of the SBP algorithm begin to control the instability and give performance comparable to the CEMB algorithm. As was previously found for shifted gauge wave tests with periodic boundary conditions [11], constraint damping [19] introduces oscillations with unacceptably large error and does not appreciably suppress the instability. Nevertheless, the performance of the constrained Sommerfeld algorithms is vastly superior to the Neumann-Dirichlet constrained algorithm considered in [10], as can be seen in Fig. 8

The convergence rates for the $2 \mathrm{D}$ shifted gauge wave tests shown in Table VI indicate clean second order convergence up to $t=10$. Figure 10 exhibits the long term performance for runs with the analytic and constrained

TABLE V. Convergence rates of $\mathcal{E}\left(g_{x x}\right)$ for the $1 \mathrm{D}$ shifted gauge wave test.

\begin{tabular}{lcc}
\hline \hline ALGORITHM & $t=10$ & $t=50$ \\
\hline AEMB & 1.97 & 1.89 \\
ASBP & 2.08 & 2.34 \\
CEMB & 1.90 & 1.97 \\
CSBP & 2.08 & 2.25 \\
\hline \hline
\end{tabular}

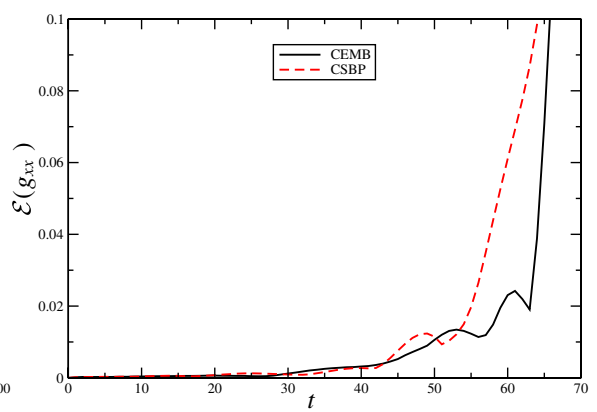

FIG. 6 (color online). Plots of $\mathcal{E}\left(g_{x x}\right)$ vs $t$ for the 2D gauge wave tests. The left graphs compare the error for the analytic SBP and embedded algorithms. The right graphs, which compare the error in the constrained SBP and embedded algorithms, exhibit the excitation of the long wavelength instability. 

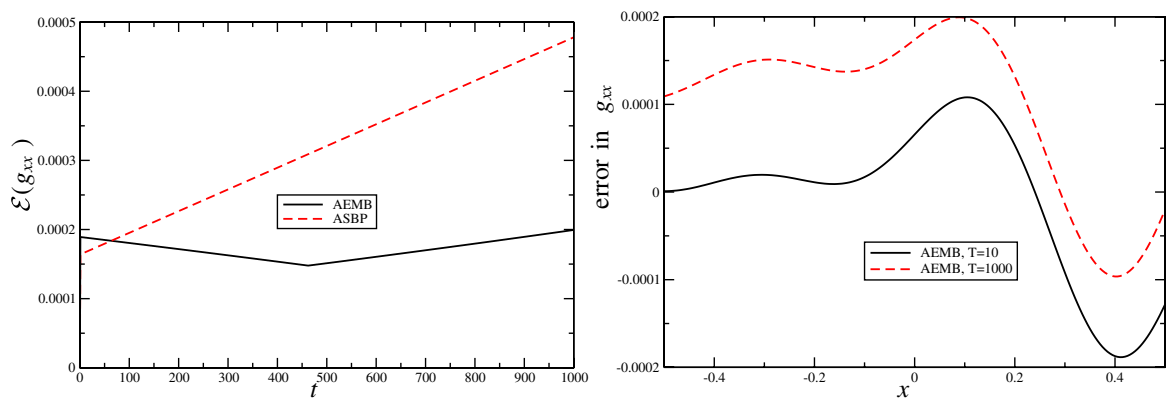

FIG. 7 (color online). Performance of the analytic SBP and embedded algorithms for the 1D shifted gauge wave. On the left, the error norm $\mathcal{E}\left(g_{x x}\right)$ is plotted vs $t$. On the right, snapshots of the error for the AEMB algorithm with the $\rho=4$ grid are shown at $t=10$ and $t=1000$. The error is long wavelength, consisting of two peaks. The overall rise in the profile is due to low level excitation of the long wavelength instability (4.14).

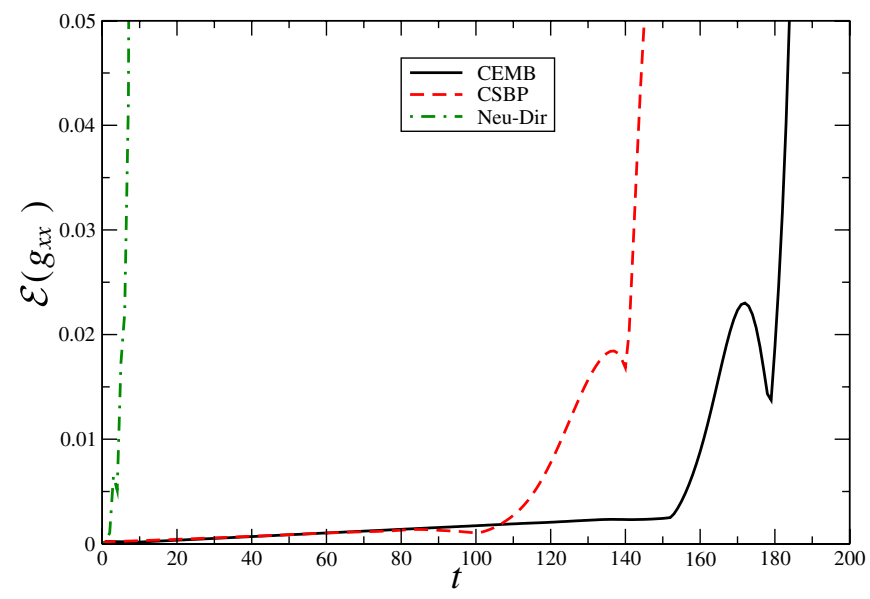

FIG. 8 (color online). Plot of $\mathcal{E}\left(g_{x x}\right)$ vs $t$ for the $1 \mathrm{D}$ gauge wave with shift simulations for the constrained CEMB and CSBP algorithms, with numerical dissipation $\epsilon_{T}=0.001$ and constraint adjustment with $b=1$, and for the Neumann-Dirichlet constrained preserving algorithm. The comparison shows that both Sommerfeld constrained algorithms clearly outperform the Neumann-Dirichlet constrained algorithm.

SBP and embedded algorithms. For the analytic algorithms (left plot), both the ASBP and the AEMB algorithms maintain excellent accuracy for 1000 crossing times. This is one of the few cases where the error in the embedded
TABLE VI. Convergence rates of $\mathcal{E}\left(g_{x x}\right)$ for the 2D shifted gauge wave test.

\begin{tabular}{lccc}
\hline \hline ALGORITHM & $t=1$ & $t=5$ & $t=10$ \\
\hline AEMB & 2.00 & 2.01 & 2.03 \\
ASBP & 2.00 & 2.02 & 2.05 \\
CEMB & 2.00 & 2.00 & 1.97 \\
CSBP & 2.00 & 2.00 & 2.04 \\
\hline \hline
\end{tabular}

algorithm is larger than the error in the SBP algorithm. This results from the 2D interpolation error introduced by the embedded algorithm. For the constrained algorithms (right plot), the long wavelength instability is excited earlier by the CSBP algorithm, which crashes at $t \approx 22$, while the CEMB algorithm runs up to $t \approx 35$. Efforts to control the instability by constraint adjustment and numerical dissipation had insignificant effect in prolonging the runs.

\section{CONCLUSION}

The preceding tests involved linearized wave, gauge wave, and shifted gauge wave metrics for which the exact solutions provide the correct boundary data. The results provide some definitive conclusions. First, the results show that constraint-preserving Sommerfeld boundary conditions give good long term accuracy, which is vastly supe-
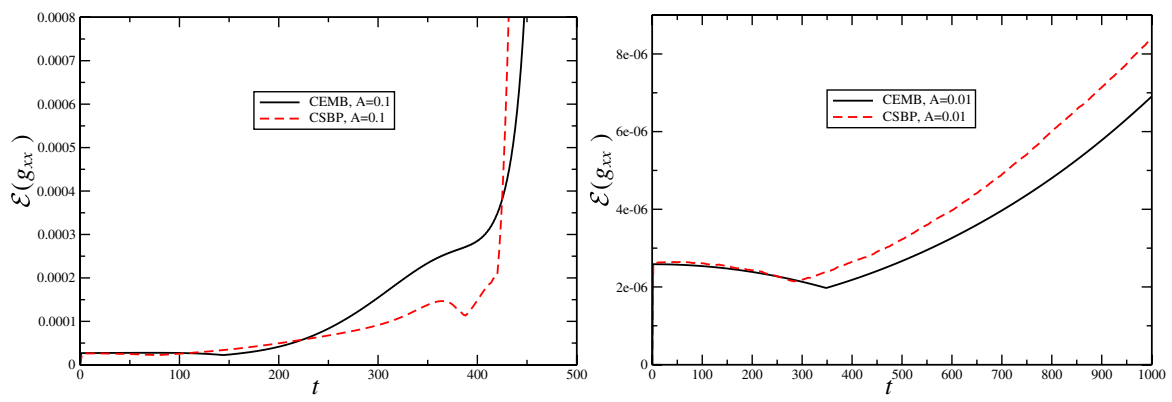

FIG. 9 (color online). Plots of $\mathcal{E}\left(g_{x x}\right)$ vs $t$ for lower amplitude simulations of the 1D gauge wave with shift simulations for the constrained versions of the SBP and embedded algorithms. The nonlinear nature of the stability is evident. 

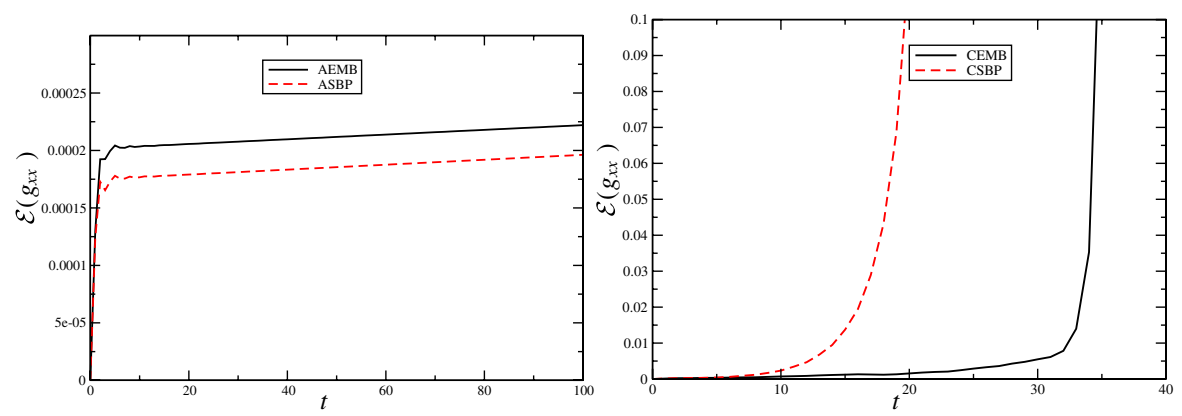

FIG. 10 (color online). Plots of $\mathcal{E}\left(g_{x x}\right)$ vs $t$ for the $2 \mathrm{D}$ gauge wave with shift. The left graphs compare the analytic SBP and embedded algorithms and the right graphs compare the constrained algorithms.

rior to previous shifted gauge wave test results for constraint-preserving Dirichlet-Neumann conditions.

Of foremost importance, the analytic versions of both the SBP and embedded algorithms clearly outperformed the constrained versions. The knowledge of the exact Sommerfeld boundary data avoids the need for computing constraint-preserving boundary conditions. One might have expected numerical noise to generate constraint violating error which the analytic algorithms could not properly handle. While this undoubtedly occurs, the analytic algorithms nevertheless outperform the constrained algorithms because the prime source of error is due to the long wavelength instabilities inherent in the nonlinear test problems. The additional error introduced by computing constrained Sommerfeld data leads to earlier excitation of these long wavelength instabilities.

Also of importance, our tests results show no clear advantage of either the SBP or embedded treatments of the boundary condition. In the more complicated case of a curved boundary, both approaches are being developed by their advocates in the computational mathematics community.

In a realistic problem, such as the binary black hole problem, a global scheme is necessary to provide physi- cally correct outer boundary data, either by using hyperboloidal time slices to extend the Cauchy evolution to infinity (see [22,23] for reviews) or by matching to an exterior characteristic or perturbative solution (see [24] for a review). Harmonic evolution offers important advantages for Cauchy-characteristic matching (CCM) which have led to successful matching in the linearized regime [10]. Primarily, the harmonic constraints can be enforced by propagating the Cauchy coordinates on the characteristic grid. This provides the proper Jacobian for injecting the Cauchy boundary data from the characteristic solution. The results of this paper should supply helpful guidance for extending the harmonic version of CCM to the nonlinear problem.

\section{ACKNOWLEDGMENTS}

We have benefited from the use of the Cactus Computational Toolkit (http://www.cactuscode.org). We thank Ian Hawke for his help in utilizing the Cactus boundary routines. Computer time was provided by the Pittsburgh Supercomputing Center. This work was supported by the National Science Foundation under Grant No. PH-0553597 to the University of Pittsburgh.
[1] B. Gustafsson, H.-O. Kreiss, and J. Oliger, Time Dependent Problems and Difference Methods (WileyInterscience, New York, 1995).

[2] H.-O. Kreiss, Commun. Pure Appl. Math. 23, 277 (1970).

[3] H.-O. Kreiss and J. Lorenz, Initial-Boundary Value Problems and the Navier-Stokes Equations (Academic, New York, 1989), reprinted as SIAM Classic, (2004).

[4] H.-O. Kreiss and J. Winicour, Classical Quantum Gravity 23, S405 (2006).

[5] F. Pretorius, Classical Quantum Gravity 22, 425 (2005).

[6] F. Pretorius, Phys. Rev. Lett. 95, 121101 (2005).

[7] F. Pretorius, Classical Quantum Gravity 23, S529 (2006).

[8] L. Lindblom, M. A. Scheel, L. E. Kidder, R. Owen, and O. Rinne, Classical Quantum Gravity 23, S447 (2006).
[9] H. Friedrich and G. Nagy, Commun. Math. Phys. 201, 619 (1999).

[10] B. Szilágyi and J. Winicour, Phys. Rev. D 68, 041501 (2003).

[11] M.C. Babiuc, B. Szilágyi, and J. Winicour, Classical Quantum Gravity 23, S319 (2006).

[12] M. C. Babiuc, B. Szilágyi, and J. Winicour, Phys. Rev. D 73, 064017 (2006).

[13] M. C. Babiuc, B. Szilágyi, and J. Winicour, Analytical and Numerical Approaches to Mathematical Relativity, Lecture Notes in Physics Vol. 692 (Springer, Berlin/ Heidelberg, 2006), p. 251.

[14] H.-O. Kreiss and N. A. Petersson, SIAM J. Sci. Comput. 27, 1141 (2006). 
[15] H.-O. Kreiss, N. A. Petersson, and J. Yström, SIAM J. Numer. Anal. 42, 1292 (2004).

[16] M. Alcubierre et al. (AppleswithApples Alliance), Classical Quantum Gravity 21, 589 (2004).

[17] "Toward standard tests for the initial/boundary problem in numerical relativity," www.appleswithapples.org.

[18] H. Friedrich, Classical Quantum Gravity 13, 1451 (1996).

[19] C. Gundlach, J. M. Martin-Garcia, G. Calabrese, and I. Hinder, Classical Quantum Gravity 22, 3767 (2005).
[20] R. M. Wald, General Relativity (University of Chicago, Chicago, 1984)

[21] O. Rinne, Classical Quantum Gravity 23, 6275 (2006).

[22] S. Husa, Lecture Notes in Physics Vol. 617 (Springer, Berlin/Heidelberg, 2003), p. 159.

[23] J. Frauendiener, Living Rev. Relativity 7, 253 (2004), http://www.livingreviews.org/lrr-2004-1.

[24] J. Winicour, Living Rev. Relativity 8, 10 (2005), http:// www.livingreviews.org/lrr-2005-10. 